\title{
Diseño, Desarrollo e Implementación de un Curso en Línea del Módulo de Fisiología Endócrina
}

\section{Design, Development and Implementation of an Online Course of the Endocrine Physiology Module}

\author{
Bertha Prieto Gòmez ${ }^{1}$,Beatriz Georgina Montemayor Flores ${ }^{2}$ \\ prieto@unam.mx, betyg@unam.mx \\ ${ }^{1}$ Fisiología \\ Universidad Nacional Autónoma de México \\ Cd. de México, México
}

Resumen- Los espacios universitarios son espacios académicos diferentes a aquellos por los cuales se transitó hace algunas décadas. El desarrollo de las Tecnologías de la Información al final del siglo $\mathrm{XX}$ ha transformado aspectos de la vida, provocando cambios en las prácticas educativas. A partir del 2010 el modelo educativo de la licenciatura de médico cirujano de la Facultad de Medicina UNAM es un modelo de currículo mixto por asignaturas con enfoque por competencias. Las características de los estudiantes demandan nuevos modelos educativos. Con el propósito de favorecer y consolidar el conocimiento básico en Fisiología Endócrina se desarrolló un curso en línea constituido por 12 sesiones en las cuales se incorporaron presentaciones de Power Point, Podcast, videos y ejercicios de autoevaluación. El curso desarrollado se encuentra disponible para los alumnos en la dirección electrónica http://fmel.facmed.unam.mx/29/moodle/login/index.php alojado en el servidor de la Facultad de Medicina UNAM. La implementación del curso en línea fue bien recibida por los estudiantes, quienes trabajaron durante las 12 sesiones. En base a los datos obtenidos se propone; hacer obligatorio el uso del curso para favorecer el rendimiento académico de los estudiantes.

Palabras claves Fisiología Endócrina, Curso en línea, Educación Médica.

Abstract- University spaces are academic spaces different from those for which one went through a few decades ago. The development of Information Technologies at the end of the 20th century has transformed aspects of life, causing changes in educational practices. As of 2010, the educational model of the surgeon's degree from the School of Medicine UNAM is a model of a mixed curriculum by subjects with a focus on competencies. The characteristics of the students demand new educational models. In order to favor and consolidate the basic knowledge in Endocrine Physiology, an online course was developed consisting of 12 sessions in which Power Point presentations, Podcast, videos and self-assessment exercises were incorporated. The course developed is available to students at the email address http://fmel.facmed.unam.mx/29/moodle/login/index.php hosted on the server of the Faculty of Medicine UNAM. The online course implementation was well received by the students, who worked during the 12 sessions. Based on the data obtained, it is proposed; make the use of the course compulsory to favor the academic performance of the students. Keywords Endocrine physiology, Online course, Medical
Education.

\section{INTRODUCCIÓN}

La educación en línea puede ser conceptualizada como una modalidad de aprendizaje en donde tanto los contenidos como las actividades, la comunicación de los participantes y la plataforma tecnológica, se realiza mediante dispositivos tecnológicos conectados a Internet (Fernández y Vallejo, 2014).

El desarrollo de internet y los avances tecnológicos han favorecido la creación de entornos de información y comunicación libres, no sujetos a un medio físico, espacios virtuales, que permiten la transmisión de la información de modo instantáneo y a nivel global, incrementando las posibilidades de aplicación en los contextos educativos (Salas y Organista, 2007).

Actualmente en los ámbitos educativos se considera que la educación en línea como un área de oportunidad para ampliar la cobertura de la educación universitaria, numerosas universidades públicas como privadas ofrecen cursos en línea apoyados de las Tecnologías de la Información y Comunicación (Tapia 2007).

Sin embargo, el uso de las TIC o de los Recursos Multimedia, por si solos no garantiza una mejora instantánea del proceso de enseñanza y aprendizaje de los estudiantes o usuarios, así como Rodríguez (2014) afirma, el diseño inadecuado de modelos educativos que involucran el uso de las TIC y o los Recursos Multimedia no mejoran el aprendizaje, al contrario, lo empeoran. Un buen uso pedagógico y didáctico de estas herramientas pueden ayudar y estimular a los alumnos, mientras un diseño deficiente o inadecuado puede confundirlos.

Evaluación de la motivación y el aprendizaje de los alumnos en los cursos en línea.

La motivación, generalmente, es vista como la aplicación de una fuerza externa o interna que induce a ejecutar una acción para obtener algo agradable o para evitar algo desagradable, todo el resultado académico de un estudiante ya 
sea eficiente o deficiente, está fuertemente dirigido por las motivaciones. (Sesento, y Lucio R. 2015)

Los alumnos que carecen de una motivación adecuada tienden a dilatar en el tiempo el momento de ponerse a trabajar, se concentran menos, estudian con menor frecuencia $\mathrm{y}$ de una forma más superficial y suelen rendirse primero ante aquellas dificultades con las que se van encontrando, lo cual, sin duda repercute en su proceso de aprendizaje y en el resultado obtenido (Tapia, 2001).

Los estudiantes pueden percibir la realización de estos trabajos como desmotivador si únicamente se plantean como actividad de aprendizaje y no tienen posteriormente repercusión en la evaluación final (Tapia 1999).

Algunos autores han estudiado el factor motivación de los alumnos que participan en los cursos en línea, en la investigación realizado por Burkle (2011), los participantes mostraron una gran motivación incluso si los estudiantes no estaban familiarizados con el uso de los dispositivos electrónicos, como las computadoras personales, para fines pedagógicos, argumentando que se sintieron motivados a estudiar cuando la presencia de los profesores traspasaba los límites del aula y cuando para aprender lo hicieron a su propio ritmo $\sin$ tener que esperar instrucciones del profesor, resultados similares se presentaron en la investigación realizada por la agencia BECTA (British Educational Communications and Technology Agency), donde concluyeron que la motivación de los estudiantes fue un factor determinante para el éxito de la implantación de las tecnologías Web 2.0.

Para evaluar la motivación de los estudiantes se han elaborado cuestionarios basados en los supuestos teóricos de la Teoría de la Orientación a Metas relacionadas con el Logro Tapia (2007)

De acuerdo con esta teoría, los alumnos se orientan en distinto grado a tres tipos de metas que definen otras tantas orientaciones motivacionales; a) Orientación al aprendizaje, que se manifiesta cuando la atención el alumno se centra regularmente en la adquisición de nuevas o mejores competencias y de los conocimientos relevantes para sostenerlas.

b) Orientación al resultado, cuando el alumno busca no tanto aprender cómo conseguir demostrar públicamente su valía y que los demás le evalúen positivamente.

c) Orientación a la evitación, que se manifiesta cuando se teme una valoración negativa de la propia valía. La introducción debe contener las mejoras que presenta respecto al estado del arte y/o conocimiento que se aporta al estado del arte.

La evaluación del aprendizaje de sus alumnos es otro de los grandes retos que los profesores y las instituciones educativas deben enfrentar, especialmente cuando la evaluación se dirige a determinar si es eficaz el aprendizaje en línea.

Una revisión sistemática realizada en 2007, entre más de 250 que evaluaban el uso del aprendizaje en línea, 126 estudios confirmaron que el aprendizaje en línea es eficaz, que los alumnos al enfrentar los cursos en línea aprenden igual que en los ambientes presenciales (Cook, Levinson, Garside, 2007).
No parece existir unanimidad acerca del concepto de evaluación. Mientras que para unos docentes se traduce en realizar una medición, para otros se trata de llevar a cabo una comprobación de los objetivos o de los resultados (Álvarez, González y García 2015), Rodríguez (2005) en cambio afirma que se entiende por evaluación, en sentido general, al conjunto de procesos sistemáticos de recogida, análisis e interpretación de información válida y fiable, que en comparación con una referencia o criterio nos permita llegar a una decisión que favorezca la mejora del objeto evaluado.

Frecuentemente en la educación en línea, la evaluación ha recibido poca atención, regularmente en estos ambientes virtuales de aprendizaje se observa una tendencia hacia formas tradicionales de evaluación, provocando una falta de coherencia entre los niveles de aprendizaje esperados en los objetivos y las actividades de aprendizaje propuestas y el proceso de evaluación que se realiza.

El proceso de evaluación debe diseñarse y desarrollarse considerando que la educación en línea promueve el papel activo del alumno como protagonista en la construcción de significados y conocimiento y la solución de problemas en contextos auténticos, características orientadas al enfoque constructivista, basado en problemas, en aprendizaje situado, entre otras.

\section{Objetivo General}

Diseñar un curso online orientado en el Aprendizaje Basado en Problemas y medir el impacto de éste en la motivación y el aprendizaje de los alumnos.

\section{Población}

La población estudiada fueron 35 estudiantes. 13 hombres, 23 mujeres, edades comprendidas entre19 y 28 años, con promedio de edad de 20 , del segundo año de la licenciatura en medicina, los cuales integraban uno de los 40. grupos de la asignatura de Fisiología, alumnos con suficiente experiencia en entornos y en educación en ambientes virtuales de aprendizaje, el $100 \%$ de los alumnos tenían experiencia en el uso de dispositivos electrónicos el 38\% había tomado cursos en línea, el 100\% había utilizado Aulas Virtuales y el 100\% estaba familiarizado con el uso de Recursos Multimedia como apoyo en el proceso enseñanza aprendizaje.

\section{Contexto}

\section{Asignatura Fisiología.}

La asignatura de Fisiología en la licenciatura en Medicina de la Facultad de Medicina UNAM forma parte de las denominadas ciencias básicas y se imparte durante el segundo año de la licenciatura en Medicina.

El curso presencial de Fisiología se desarrolla durante 35 semanas, el módulo de Fisiología Endócrina se imparte durante 10 semanas. Debe contener la necesidad de su realización, objetivos, contexto y público objetivo.

El programa académico de la asignatura se divide en tres Unidades; $1^{\mathrm{a}}$. Fisiología Celular y Fisiología del Sistema Nervioso, $2^{\mathrm{a}}$ Fisiología Cardiovascular y $3^{\mathrm{a}}$ Fisiología de los Sistemas Endócrino y Digestivo. 


\section{DESCRIPCIÓN}

Con la finalidad de que un curso en línea alcance los objetivos que se propone, es indispensable que el diseño de éste se conforme de dos dimensiones indispensables, la pedagógica y subordinada a ésta la tecnológica.

El Modelo Educativo del curso en línea "Módulo de Fisiología Endócrina" se centra en el estudiante y su aprendizaje. Se desarrolló bajo el enfoque constructivista del aprendizaje, por lo que se encuentra centrado en el estudiante, considerado como un agente participativo, protagonista activo en la construcción de su propio conocimiento.

\section{Características del Modelo Educativo.}

El modelo presenta como características;

1. La flexibilidad, en cuanto que su uso supera las barreras del espacio y el tiempo, creando una dimensión en donde no importan los horarios de los estudiantes, donde vivan ni cuales sean sus condiciones sociales y responsabilidades Debe familiares, el modelo ofrece a todos la posibilidad de estudiar.

2. Personalizado, al reconocer que existen diferentes ritmos y tiempos para aprender.

3. Interactivo ya que ofrece actividades en entornos virtuales de enseñanza y aprendizaje

El diseño del curso en línea incorporó las siguientes metodologías;

1.Diseño Instruccional Basado en los principios fundamentales de Merrill, aplicado al diseño de los ambientes de aprendizaje y se enriqueció con las propuestas instructivas de la enseñanza situada, la cual promueve que el alumno aprenda enfrentando las mismas situaciones que afrontan los expertos del área de estudio, en este caso en particular las actividades referidas a la práctica de la medicina y del aprendizaje significativo, aprendizaje en donde el alumno relaciona de manera sustancial la nueva información con sus conocimientos y experiencias previas, Ausbel (1976). Entre las estrategias de aprendizaje significativo centradas en el aprendizaje situado seleccionamos; 1 . Análisis centrado en la solución de problemas auténticos, 2. El aprendizaje en situaciones reales y 3. El aprendizaje mediado por TIC's.

2.Análisis Cognitivo de Tareas (Castañeda, 2002), para el análisis del contenido temático, diseño y desarrollo de los módulos para el procesamiento de la información, las actividades de aprendizaje, los ejercicios de autoevaluación, y los ejercicios de aprendizaje basado en problemas.

4.Modelo de autorregulación de Pintrich (2000), en el desarrollo de la meta pedagógica referida a la integración de la enseñanza del aprendizaje autorregulado, junto con el aprendizaje de los contenidos temáticos, técnicas y estrategias aplicadas en tareas auténticas.

5.Diseño Centrado en el Usuario, aplicado a la selección, diseño y desarrollo de los materiales didácticos digitales interactivos.

El curso en línea comprendió los contenidos temáticos del Módulo FISIOLOGIA del SISTEMA ENDOCRINO, se desarrollaron 12 sesiones, las cuales se conformaron diferentes Recursos Multimedia como; Presentaciones en Power Point, Podcast, videos, artículos. Todas las sesiones presentaron ejercicios de autoevaluación.

\section{Instrumento de Evaluación.}

El curso en línea no fue una actividad obligatoria para los alumnos, solo participaron aquellos que se interesaron.

Con el propósito de medir el aprendizaje y la motivación consideramos la propuesta de Rodríguez (2005) y decidimos utilizar como criterio referencial; a. el promedio grupal que los alumnos obtuvieron en el examen departamental que incluyo el módulo que trabajaron durante el curso en línea y b. los supuestos teóricos de la Teoría de la Orientación a Metas relacionadas con el Logro, (Tapia 2007) correlacionados con los registros de participación que realiza la propia plataforma Moodle para el seguimiento de las actividades de los alumnos durante el curso en línea, las cuales se presentan en la Tabla 1, respectivamente.

Tabla 1

Correlación de los Supuestos Teóricos y actividades de los alumnos

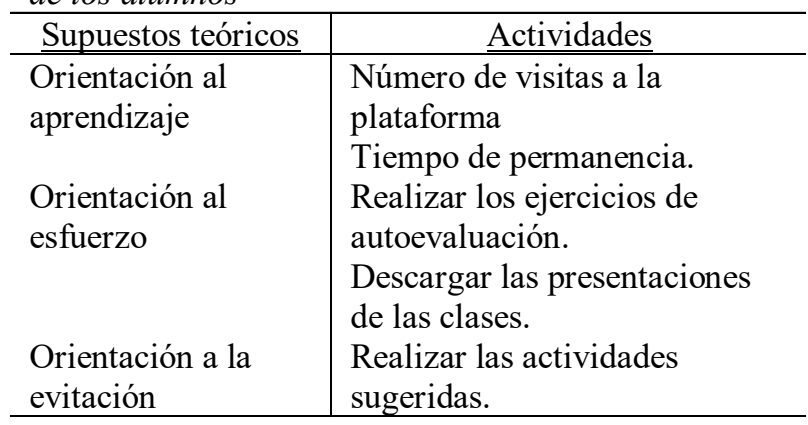

\section{Resultados}

El objetivo de este trabajo fue el diseño de un curso en línea para promover el aprendizaje de la Fisiología del Sistema Endócrino, en la Tabla 2 se presenta la distribución de los contenidos de las sesiones desarrolladas.

El curso se encuentra disponible para los alumnos en la dirección http://fmel.facmed.unam.mx/29/moodle/login/index.php Alojado en el servidor de la Facultad de Medicina UNAM.

Al término del curso los alumnos inscritos en el curso en línea presentaron el mismo examen departamental que el resto de la generación y el promedio de calificación obtenido se encontró por encima de la media del rango de promedio de la generación, lo cual nos permite proponer que el aprendizaje de estos alumnos fue equiparable con el aprendizaje de los alumnos que no utilizaron el curso en línea.

La implementación de un curso en línea como propuesta metodología para el estudio y aprendizaje del Sistema Endocrino fue bien recibida por los estudiantes, quienes trabajaron motivados y sin problemas durante las 12 sesiones que lo integraron

En base a los datos obtenidos se propone; hacer obligatorio el uso del curso en línea desarrollado para mejorar la adquisición y procesamiento de la información que se presenta en las sesiones y así favorecer el rendimiento académico de los estudiantes. 
Tabla 2

Distribución de la organización de los contenidos de las sesiones desarrolladas

\begin{tabular}{c|l}
\hline Sesión & Contenido Temático \\
\hline 1. & Presentación \\
2. & Fisiología del Sistema \\
& Endócrino \\
3. & Hipotálamo e Hipófisis \\
4. & Fisiología del \\
& crecimiento normal. \\
& Factores de crecimiento \\
5 & Tiroides \\
6 & Glándula Pineal \\
7 & Ritmos Biológicos \\
8. & Síndrome Metabólico \\
9. & Tejido Adiposo \\
10 & Páncreas Endócrino \\
11 & Gónadas Femeninas \\
12. & Gónadas Masculinas \\
\hline
\end{tabular}

El 70\% de los alumnos participaron en el curso en línea, los resultados referentes a la motivación se describen en la tabla 3 .

Tabla 3

Correlación de los Supuestos Teóricos, actividades de los alumnos

y porcentaje de participación

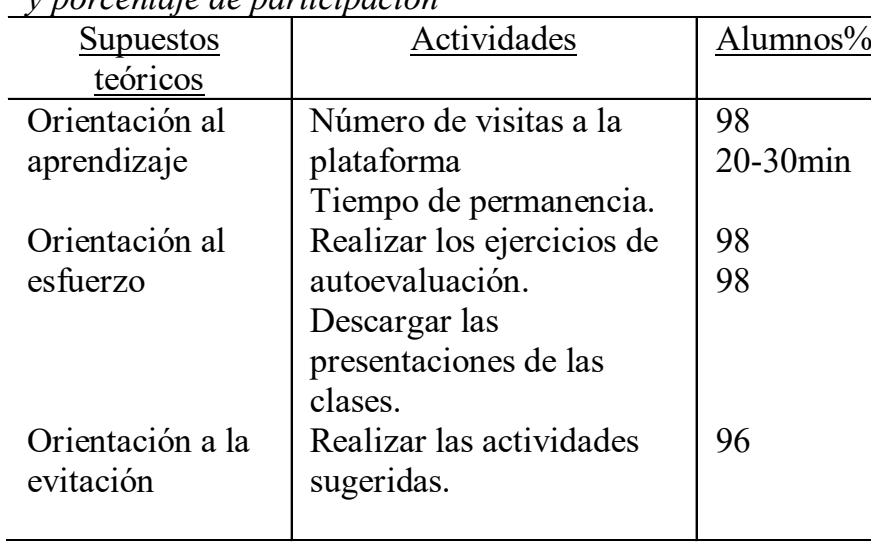

Las sesiones que más visitas recibieron fueron la $11^{\mathrm{a}}$ el $67 \%$ de los alumnos inscritos, la segunda fue la $10^{\mathrm{a}}$ y la $7^{\mathrm{a}}$ ambas con el $63 \%$, las que reportaron menos visitas fueron las sesiones $2^{\mathrm{a}} 43 \%$ y la $3^{\mathrm{a}}$ con $53 \%$. Durante la semana previa al examen del módulo de Fisiología Endócrina el 95\% de los alumnos inscritos y que presentarían el examen visitaron la plataforma del curso.

\section{CONCLUSIONES}

La implementación de un curso en línea como propuesta metodología para el estudio y aprendizaje del Sistema Endocrino fue bien recibida por los estudiantes, quienes trabajaron motivados y sin problemas durante las 12 sesiones que lo integraron
En base a los datos obtenidos se propone; hacer obligatorio el uso del curso en línea desarrollado para mejorar la adquisición y procesamiento de la información que se presenta en las sesiones y así favorecer el rendimiento académico de los estudiantes.

\section{REFERENCIAS}

Álvarez, B., González C. y García N. (2015) La motivación y los métodos de evaluación como variables fundamentales para estimular el aprendizaje autónomo. Recuperado de http://redu.net/redu/files/journals/1/articles/53/public/53-42-2PB.pdf.

BECTA (2007) Emerging Technologies for Learning, vol. 2 Recuperado de http://cent.uji.es/octeto/node/2060

Bukle, M (2011) El aprendizaje on-line: oportunidades y retos en instituciones politécnicas. Recuperado de file://C:/Users/georgina/Downloads/10.3916_C37-201102-04(1).pdf

Cook DA, Levinson AJ, Garside S. (2008) Internet-Based learning in the Health Professions: A Meta-Analysis. JAMA. Recuperado de 2008;300:11811196.

Castañeda Figueiras, S. (2004). Evaluando y fomentando el desarrollo cognitivo y el aprendizaje complejo . Psicología desde el Caribe, (13), 109-143.

Dorrego, E. Educación a distancia y evaluación del aprendizaje. Recuperado https://www.um.es/ead/red/M6/dorrego.pdf

Fernández, K y Vallejo, A. (2014) La educación en línea: una perspectiva basada en la experiencia de los países. Recuperado de http://www.cucs.udg.mx/revistas/edu desarrollo/anterior es/29/029_Fernandez.pdf

Rodríguez C., Ma. José (2005). Aplicación de las TIC a la evaluación de alumnos universitarios. Recuperado de http://www3.usal.es/ -teoriaeducacion.

Sesento, L. y Lucio R. (2015). La motivación, trabajo permanente en el aula. Recuperado de http://www.ecorfan.org/spain/researchjournals/Investiga cion_y_Desarrollo/vollnum2/8-Investigacion-ydesarrollo-128-132.pdf

Tapia, J. (1999). Motivar para el aprendizaje Teoría y estrategias Recuperado de http://www.terras.edu.ar/biblioteca/6/TA_Tapia_Unidad 4.pdf

Tapia, J. (2007). Evaluación de la motivación en entornos educativos. Recuperado de http://sohs.pbs.uam.es/webjesus/eval_psicopedagogica/le cturas/eval\%20motiv.pdf

Tapia, J. (2011). MOTIVACIÓN PARA APRENDER, MOTIVAR PARA SER. Recuperado de file://C:/Users/georgina/Downloads/DialnetConferencia-3674721.pdf 